\title{
A Mechanism for Elbow Exoskeleton for Customised Training
}

\author{
Soumya K. Manna, Venketesh N. Dubey, Member, IEEE
}

\begin{abstract}
It is well proven that repetitive extensive training consisting of active and passive therapy is effective for patients suffering from neuromuscular deficits. The level of difficulty in rehabilitation should be increased with time to improve the neurological muscle functions. A portable elbow exoskeleton has been designed that will meet these requirements and potentially offers superior outcomes than human-assisted training. The proposed exoskeleton can provide both active and passive rehabilitation in a single structure without changing its configuration. The idea is to offer three levels of rehabilitation; namely active, passive and stiffness control in a single device using a single actuator. The mechanism also provides higher torque to weight ratio making it an energy efficient mechanism.
\end{abstract}

\section{INTRODUCTION}

Patients suffering from stroke and other neuromuscular diseases usually lose their muscle functions. Such occurrences may lead to loss of power or complete paralysis of limbs if left unused in the acute phase. It is recommended that intensive occupational therapy in the early stages [1] may provide a superior rehabilitation to affected limb. However, this requires dedicated time for therapy, supervised training and controlled routine for the early recovery which is generally not tenable due to therapist fatigue, time, cost and repeatability requirements of the rehabilitation process. Present annual health and social costs of caring for disabled stroke patients are estimated to be in excess of $£ 5$ billion in the UK [2]. Also, it is difficult for the patients to receive the recommended amount of therapy manually [3]. If a portable exoskeleton could be designed that will meet these requirements, it could potentially offer superior outcomes than the human-assisted training which is currently in use.

Shoulder and elbow joints are mainly responsible for gross positioning of the upper arm during pick and place activities of daily living [4]. The aim of this research is to design a mechanism to allow a variety of elbow rehabilitation training in a single module since it is one of the simplest human joints and majority of gross manipulation tasks cannot be performed without the elbow motion [4]. In the simplest form, it could be visualized as a revolute joint with one degree of freedom with the further flexibility provided by pronation-supination motion of forearm. During the last two decades, a large number of arm exoskeletons have been developed and a lot of technical research articles have been published [5, 6]. Out of these, most are platform-based systems which might be the reason for not adopting exoskeletons for rehabilitation on a large scale. Despite the potential benefits provided by the stationary arm exoskeletons, stroke patients and healthcare professionals

Soumya K. Manna and Venketesh N. Dubey are with Faculty of Science and Technology, Bournemouth University, Fern Barrow, Poole, UK (corresponding author phone: 44-1202-965986; fax: 44-1202-965314; email: vdubey@bmth.ac.uk). would prefer a compact, user-friendly and portable system [7]. Out of few commercially available portable exoskeletons in the market, majority of them are based on the elbow joint [5]. Therefore, development of a portable elbow exoskeleton needs to be given a due consideration for arm rehabilitation.

\section{REHABiLitation StRATEgy AFTER STROKE}

There is a proper process to recover from the acute phase to full recovery stage. According to Brunnstrom Approach [8], seven sequential stages have been introduced after stroke to regain the controlled muscle function in the view of spasticity and involuntary muscle movement. After thoroughly revising all the seven stages of rehabilitation it can be distinctly put into three stages as shown in Fig.1.

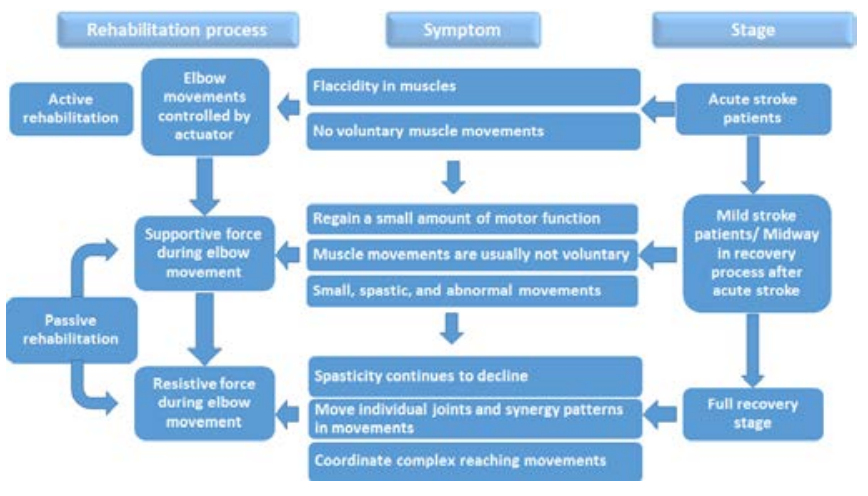

Figure 1. Three phases of rehabilitation process after stroke

From a recovery standpoint exoskeleton should provide all kinds of motions required for all three stages. Signs of recovery can be ascertained from the symptoms at different stages. In the acute phase, there is no muscle movement due to lack of coordination between muscle and brain. This is called flaccidity which does not allow the patient to make voluntary muscle movement due to nerve damage. Patient in this stage cannot even initiate any joint movement. If this situation remains for a long time without undergoing any therapy, the muscle becomes weaker and may cause complete paralysis. Therefore, the muscle needs to be put in to action by applying external force using exoskeleton. Here human joint follows a defined trajectory throughout the active range of motion and can be controlled by an exoskeleton. This also helps to reduce spasticity which occurs after acute stage. The second stage of recovery process shows a much better condition where spasticity continues to decrease and patient regain small muscle function which helps to do some movement though it is not voluntary. This phase requires a passive supportive force to reach to the desired position and orientation. Exoskeleton providing passive rehabilitation can be helpful to reduce the abnormality in the movement. It also helps to generate complex coordinated muscle control in the 
upper limb. Repetitions of daily activities improve the controllability of muscle. In full recovery stage, spasticity completely disappears; the isolated joint movement seems to be getting better in the areas affected by stroke. The patient is able to initiate complex voluntary movement but not with enough force. Therefore joints are still experiencing motor deficit which can be supported to improve neuro-motor functions.

\section{EXISTING ELBOW EXOSKELETONS}

There have been many attempts to develop portable elbow exoskeletons; a few of them have been brought out for direct patient applications. However, these exoskeletons focused on one aspect of the design but failing on providing standard rehabilitation training because either those are active actuator based or passive system based on spring or rubber band [5, 6]. Some are emphasising on portability and mechanical advantage whereas other are targeting on the standalone rehabilitation process. There is hardly a single exoskeleton providing all types of exercises required for the three phases of rehabilitation as shown in Fig 1. Titan arm [9] is a commercially available portable elbow joint exoskeleton which is based on non-localized actuation using a cable drive and a ratchet-based braking system. In this design, actuators along with energy source are located on the backpack to reduce the load on a human arm. ULERD [10] is a compact elbow exoskeleton which provides upper-limb resistance training using HMI (Human machine interface). Myomo [11] is an arm exoskeleton based on active actuator where a sophisticated control system can measure the patient's intention of movement using different biosensors and can generate variable motor torque on the patient's effort. This type of device can be useful as the assistive device but not suitable for rehabilitation training. In software based solution, exoskeleton may perform a discordant behaviour on sudden impact force due to delay. Continuous engagement of electric motor along with other electronic components results in constant draining of energy. This technique is not suitable for an energy efficient mechanism. Also, the human joint motion is always under motor control which might not be a good idea from safety point of view. If the motor moves beyond the anatomical limit of the human joint due to malfunction an accident may happen. A passive exoskeleton is developed based on an elbow-forearm interlock joint mechanism to coordinate two movements of elbow joint for the patients suffering from tremor [12]. In this mechanism, two DOF of elbow joint are merged into a single passive one. An upperlimb power-assist exoskeleton is developed based on elbow joint actuated by pneumatic artificial muscle [13]. However, it has a series of problems like lower bandwidth, non-linear characteristics, unidirectional operation and bigger size because it uses pneumatic artificial muscle as the actuator. NEUROEXOS [14] is a neurobotics elbow exoskeleton for post-stroke physical rehabilitation but it has a big hydraulic cylinder connected to it for antagonistic actuation. Such actuators may be effective for rehabilitation but not for a portable system. Pneumatic bending joint using skewed rotary elastic chamber (sREC) is used in a soft elbow trainer to assist patient for safe and effective rehabilitation [15]. It has a pneumatic elastic chamber which allows self-alignment to the polycentric movement of the human joint axis.
Harmonic drive [16] based exoskeleton is used for rehabilitation although the cost of the actuator appeared to be very high. A bidirectional elbow exoskeleton device is developed based on rotationally twisted string actuator. This mechanism consists of antagonistic control for elbow movement [17]. WREX [18] is based on one of simplest designs where the rubber band is used to reduce antigravity torque during elbow movement. It is a passive system but useful for the children with neuromuscular disabilities.

\section{WORKING PRINCIPLE OF THE PROPOSED ELBOW EXOSKELETON}

The aim of the proposed exoskeleton is to provide four independent properties which may be considered to be the main requirements for an exoskeleton to provide standard rehabilitation training.

- To enhance the functional neuroplasticity using active and passive rehabilitation process in a single system.

- To vary joint stiffness to change its resistance during therapy depending on the task requirement.

- To develop a gravity compensating mechanism adjustable to different joint torques.

- To design an intelligent mechanism capable of giving higher torque to weight ratio compared to existing models.

Active rehabilitation is required to provide rehabilitation to acute stroke patients and passive rehabilitation will be needed for mild stroke patients as well as for those who are undergoing recovery process. It may be advantageous to have both characteristics in a single exoskeleton by compensating their shortfalls. In the proposed exoskeleton, both active and passive rehabilitation process has been introduced in a single structure without using any extra actuator. This technique will help utilize the same motor to carry out different modes of training.

An innovative elbow joint mechanism is developed to enhance the torque to weight ratio. After a thorough literature review [6], it has been found that most of the elbow joint exoskeletons designed or commercially available have motors directly connected to its joint. Since exercises during active rehabilitation are executed at different load, motors are designed with certain characteristics to match specific speedtorque requirement. When the exoskeleton attempts to lift the human arm it is attached to, against the gravity (including its own weight), it is subjected to a varying amount of torque. As those exercises have been carried out by the external motor, it must be able to develop enough torque to start, accelerate and operate the exercises at the rated speed. Motor providing comparatively higher torque is bigger in size and heavier in weight. The problem occurs when a heavy and bulky DC motor is located at the joint axis which needs to be carried out by the patient. Also for other properties such as stiffness control and compensating for gravitational force, these exoskeletons would need more actuators and additional components which would make it heavy and difficult to be used by patients. Joint actuation in human arm takes place 
due to ligaments which behave like tendon and provide stability to joint. Biceps muscle helps the forearm for elbow flexion and triceps muscle applies opposite motion -extension to straighten the forearm back to its own position. The pattern of human joint movement requires a lower amount of torque compared to a motor connected at joint. Therefore, the developed mechanism is based on the biomechanical structure of human elbow joint.

The working principle of this exoskeleton has been based on the standard rehabilitation process which requires a few sequential stages for a specific type of rehabilitation. As per the standard rehabilitation protocol, people have to go through active rehabilitation in the first stage of training after that the patients have to undergo passive rehabilitation to assist their own movements during the exercises. The last stage requires that the variety and difficulty of the exercises should be changed by changing the joint stiffness. All these steps are not being performed at the same time and are followed gradually according to the status of the patient. Accordingly, the proposed exoskeleton provides certain regions in the structure offering the specific rehabilitation training. The idea is to divide the whole working region into three sub-regions consisting of different training regime dedicated to providing specific exercises. All these regions are inter-connected and will appear one after another automatically. Therefore, the patients don't need to change the exoskeleton or press any switch to achieve the much needed optimal training. A couple of springs (compression) have been used in the exoskeleton to switch between different regions using the stiffness property of the springs. No extra energy source is required to move from one training regime to another making it an energy efficient mechanism. The other advantage of this design is that a single motor is used to achieve all the above features. The three regions of rehabilitation are shown in Fig. 2, the mode of operation in each region is distinct from one another.

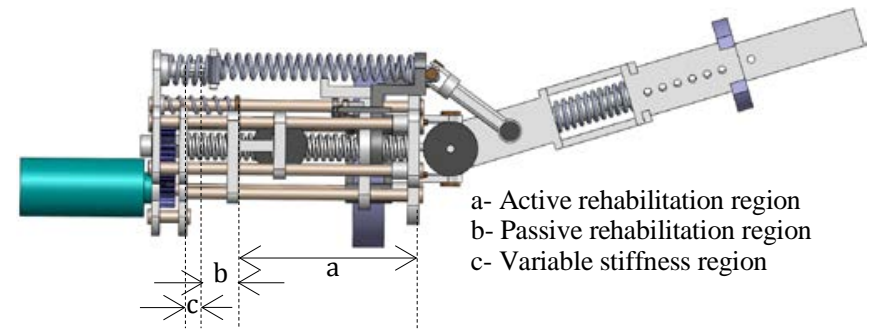

Figure 2. Active, passive and variable joint stiffness regions

\section{A. Free arm movement in active rehabilitation region}

At the acute stage of stroke, the patient does not have any control on their arm. Therefore, they are being rehabilitated with free movement consisting of some predefined orthopaedic lessons prescribed by an expert. The patient does not have any active participation; these exercises are totally controlled by the exoskeleton system. These movements are mostly restricted to isolateral exercises which are modified and can include some activities of daily living. Also, the user is able to carry out all exercises modules at a different speed, as a result, the exercises can be operated at different frequency level which could be beneficial for the patient.

\section{B. Assisting force in passive rehabilitation region}

After the primary phase patients generally get a little bit of muscle power as their neuro-motor function improves. In this phase, patients are able to move their arm but not having enough force to keep or reach out to a specified location. It's therefore required to use a support structure which would provide balancing force against gravity.

\section{Resistive force in variable stiffness region}

After a few months of continuing training patient gains some arm power. It is the time to put those patients into real life exercises. For the first two phases the force provided to the exoskeleton is always supportive but in this phase, constant resistive force is imposed to restrict the movement. Now the level of difficulty should be increased, this could be varied by changing the joint stiffness of the exoskeleton. It could help the patient in terms of recovery from resistive training. As the time passes the patient gets familiar with all training conditions so their arm function should improve.

\section{DESIGN DESCRIPTION}

In the proposed exoskeleton, the forearm is being actuated using a four-bar mechanism. The elbow exoskeleton design uses a lead-screw followed by a slider-crank mechanism (Fig. 3a). The schematic diagram of the exoskeleton along with its 3D model is shown in Fig. 3. The lead-screw joint is actuated by a motor connected at the end of the system. The motor is horizontally aligned with the structure and not occupying any extra space on either side resulting in a stable system. The electromechanical motor provides required torque during active rehabilitation whereas springs attached to the mechanism contribute to joint stiffness control as well as gravity compensation by providing static or variable assistive force during passive rehabilitation. The range of motion of elbow joint exoskeleton during active rehabilitation is 0-135 degree. Two coupled gears transfer motion from the motor to the lead-screw. The lead-screw and slider crank mechanism are not directly connected to each other. There are two guides working as slider on the leadscrew, one of which acts as a nut and the other is only concentric to the lead-screw. A spring $\left(\mathrm{S}_{6}\right)$ loaded selfactuated lock is there to hold the nut and the concentric slider to form a single unit during active rehabilitation using its two wings. As a result, those two sliders cannot come out of the locked phase. Motor controls the position of the nut slider which decides the operation of the exoskeleton as well as the region of rehabilitation. A fixed number of rotations of the motor restrict the exoskeleton in active rehabilitation region. A slight increment in the motor rotation creates a situation where $\mathrm{S}_{6}$ pushes a compression spring $\left(\mathrm{S}_{5}\right)$ connected at the reference point. Due to the higher stiffness of $S_{5}, S_{6}$ will be compressed and free the concentric slider by opening its jaws. The two jaws are connected to the nut slider using $S_{5}$ in such a way that in normal operating conditions, those two jaws always remain parallel but a small opposite force will open those jaws. Beyond this the exoskeleton enters into passive rehabilitation region and the required torque is now supported by an extension spring $\left(\mathrm{S}_{2}\right)$. This technique allows 
the exoskeleton to maintain firm contact with the elbow rotation during active rehabilitation and flexible contact in passive rehabilitation. $\mathrm{S}_{2}$ helps the elbow joint to be torque balanced at every configuration due to spring force and only a small joint torque is required to move the arm from the statically balanced condition. The above concept does not require any energy source and could hold the arm in a balanced position during passive rehabilitation. $S_{1}$ is a compression spring connected between $S_{2}$ and reference place. It can be compressed by moving the nut slider in backward direction due to further rotation of the motor, thus changing the front end of $S_{2}$ resulting different assistive force for the patients during passive rehabilitation. An additional setup of extension spring assembly $\left(S_{3}\right.$ and $\left.S_{4}\right)$ is used for changing the joint stiffness of the exoskeleton in variable joint stiffness region. Further backward movement of nut slider is used to stretch both $\mathrm{S}_{3}$ and $\mathrm{S}_{4}$, thus changing the contact force at the elbow joint. Two pairs of $S_{3}$ and $S_{4}$ are connected on the both sides of the elbow joint in parallel to maintain it in a stable condition. Part of the forearm supported structure acts as the crank in the slider-crank module which is supported on a universal joint. In spite of using a motor at the elbow joint, the forearm is actuated by the crank. This configuration is similar to that in the human elbow joint with biceps muscle. A universal joint is used to replace the normal revolute joint. One side of this universal joint is connected to forearm supporting link to provide elbow movement and the other side is used for changing the joint stiffness during different exercises. The universal joint is used to compensate a little bit of movement in sagittal plane since the rotation axis is not fixed during flexion and extension because of the structure of double quasi-conic frustum at the sagittal plane of the elbow joint. The universal joint used at elbow has two degrees of freedom; one is active and another is passive. Active joint is driven by motor cum leadscrew and is responsible for flexion-extension in vertical plane whereas small movements across sagittal plane are being taken care of by the passive joint. If the arm segment is connected rigidly it will create problem to user skin and articulation because the elbow joint behaves like a loose hinge joint. The passive joint is capable of compensating that variation laterally ( \pm 5 deg.). Two mechanical constraints with rubber padding have been added at the end of the joint structure to restrict the passive motion beyond the range. A forearm supportive link having discrete holes is attached to adjust it for all person of variable arm length as seen in Fig. 3b. There is a compression spring attached to the forearm supporting link with a sliding joint for compensating the misalignment during movement.

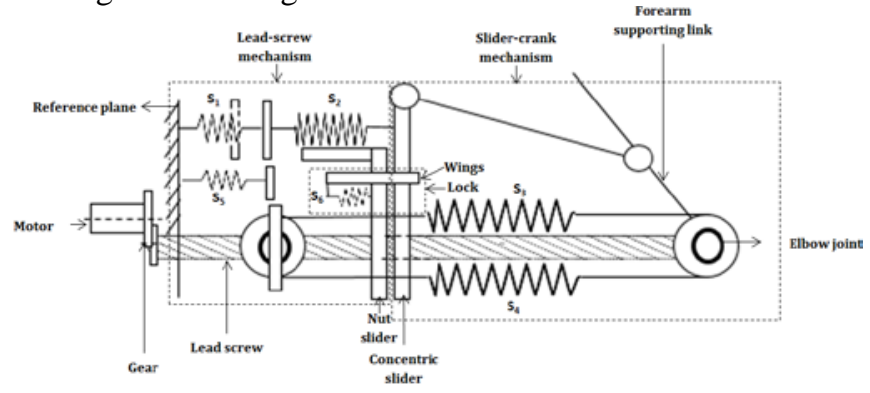

a. Schematic diagram of the exoskeleton

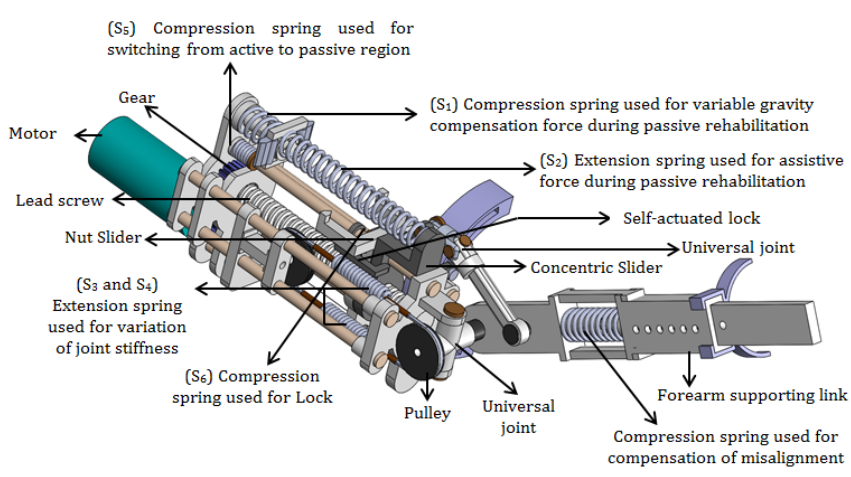

b. 3D model of the exoskeleton

Figure 3. Elbow joint exoskeleton

From the above discussion, it should be clear that all the compression and extension springs $\left(\mathrm{S}_{1}, \mathrm{~S}_{2}, \mathrm{~S}_{3}, \mathrm{~S}_{4}, \mathrm{~S}_{5}\right.$ and $\left.\mathrm{S}_{6}\right)$ are used in the exoskeleton either for giving spring force or switching from one stage of rehabilitation to another. Also, it is advantageous to have a single motor for executing all types of exercises with the help of springs. For further clarification, the specific mechanism for three stages of rehabilitation is shown in Fig. 4 with the schematic diagrams.

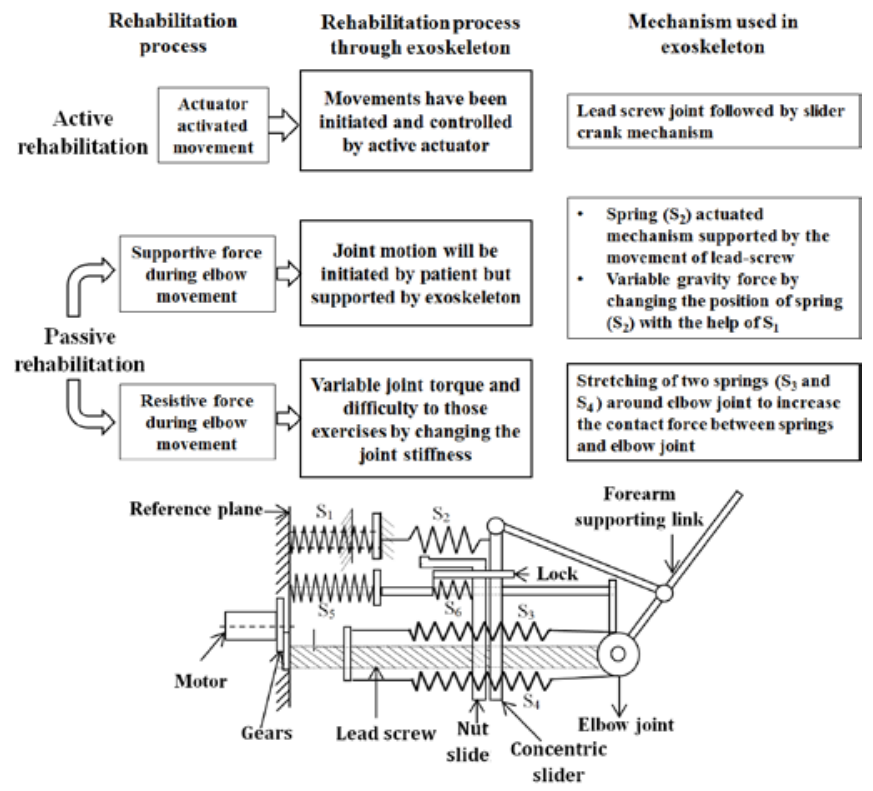

a. Exoskeleton in active rehabilitation region (Locked)

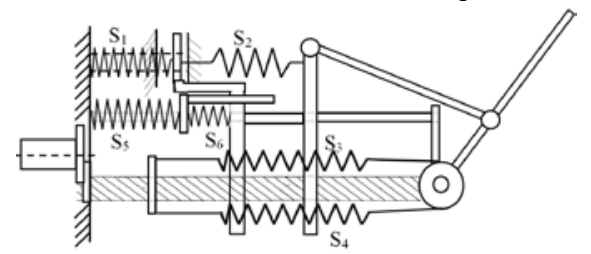

b. Exoskeleton in passive rehabilitation region (unlocked)

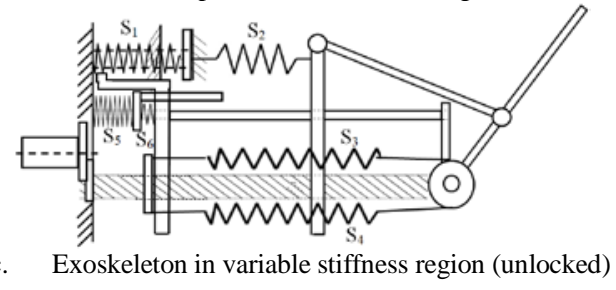

Figure 4. Elbow mechanism through specific rehabilitation processes 
$\mathrm{S}_{3}$ and $\mathrm{S}_{4}$ are normally used for the changing the joint stiffness (Fig. 5). The stiffness of both $S_{3}$ and $S_{4}$ are different because of their spring parameters. The starting position of $\mathrm{S}_{3}$ and $\mathrm{S}_{4}$ is connected to a nut slider which can change its position on the lead-screw depending upon the motor rotation.

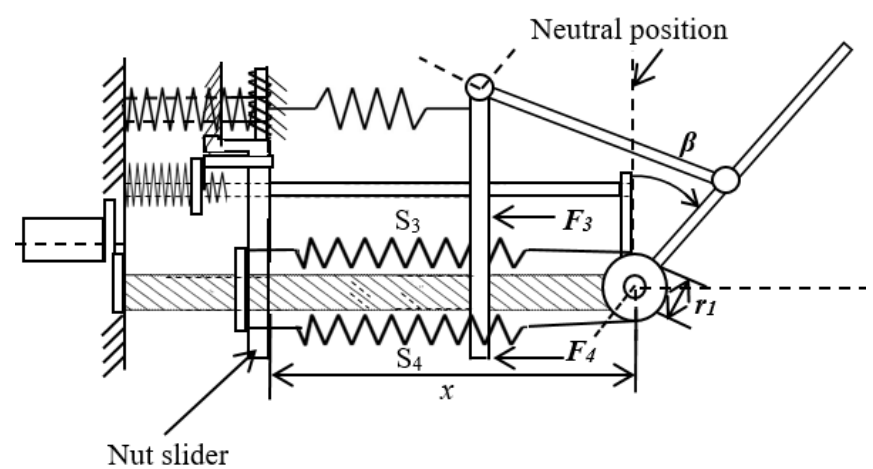

Figure 5. Elbow exoskeleton in variable stiffness region

Stiffness of the spring $\mathrm{S}_{3}=K_{3}$

Spring force exerted by $S_{3}$,

$$
F_{3}=-K_{3}\left(x+r_{1} \beta-x_{0}\right)
$$

Where $x_{0}$ is the free length of the spring $S_{3}$

Stiffness of the spring $\mathrm{S}_{4}=K_{4}$

Spring force exerted by $S_{4}$,

$$
F_{4}=-K_{4}\left(x-r_{1} \beta-x_{0}^{\prime}\right)
$$

Where $\mathrm{x}_{0}{ }_{0}$ is the free length of the spring $\mathrm{S}_{4}$

Joint torque $\tau$ is created due to differences between two spring force is given by

$$
\tau=r_{1}\left(F_{3}-F_{4}\right)
$$

The joint stiffness of the elbow joint is same as the torsional stiffness K’ given by

$$
\frac{\tau}{\beta}=\frac{r_{1}\left(F_{3}-F_{4}\right)}{\beta}
$$

Two pairs of $\mathrm{S}_{3}$ and $\mathrm{S}_{4}$ are connected in this mechanism, therefore, the force exerted by both springs will be doubled. Putting the value of $F_{3}$ and $F_{4}$ in equation (4) the elbow joint stiffness is given by

$K^{\prime}=\frac{2 r_{1}\left\{\left(K_{4}-K_{3}\right) x+\left(K_{3} x_{0}-K_{4} x \prime_{0}\right)\right\}}{\beta}-2 r_{1}^{2}\left(K_{4}+K_{3}\right)$

\section{The PRototype Design}

The prototype design is developed based on a simple idea where weight reduction was the main priority because it helps in building a portable structure. The selection of material is directly contributing to the weight of the device so the elbow exoskeleton has been produced using 3D printing. ABS (Acrylonitrile butadiene styrene) material was used which is suitable for a portable and flexible structure.
The weight of the whole system is $1.8 \mathrm{~kg}$ and the weight of the motor is $210 \mathrm{gm}$. DC motor is selected from DFROBOT. The motor is able to generate $50 \mathrm{~kg}-\mathrm{cm}$ torque at $50 \mathrm{rpm}$. Gears were made from nylon-101. The length of the module connected to the upper arm is $20 \mathrm{~cm}$ whereas width is $11 \mathrm{~cm}$ and thickness is $4.75 \mathrm{~cm}$ respectively. The forearm supporting link is $25 \mathrm{~cm}$ in length. Soft orthopaedic material polypropylene is used for holding dorsal and ventral sides of both upper and forearm. It will absorb skin moisture and provide user comforts. Holding cuff with wide area fitted to both arms can transfer uniform load to the user. Spring is made up of ASTM A228 material which is able to provide the required stiffness of joint torque. The design could be further miniaturised to make it lighter. The prototype of the elbow exoskeleton is shown in Fig. 6.

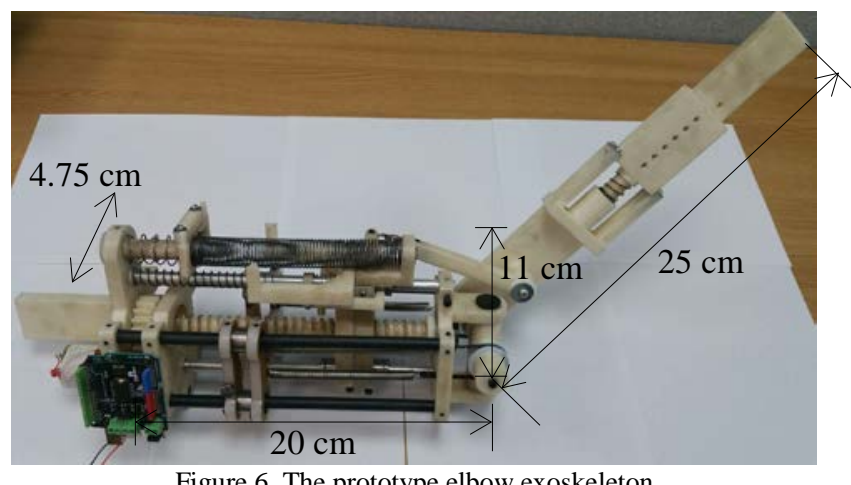

Figure 6. The prototype elbow exoskeleton

\section{Simulation RESUlts}

A simulation study on the designed exoskeleton model was performed in Solidworks ${ }^{\mathrm{TM}}$ platform using Motion Analysis tool. It includes experiments for evaluating the mechanical advantage of the design. The experiment does not include any human arm weight. Therefore, the simulation results only count the weight of the whole exoskeleton. Fig. 7 shows the comparative torque requirement for the proposed model with respect to motor placed directly at the joint during active rehabilitation. The two models are simulated at an angular velocity of $0.63 \mathrm{rad} / \mathrm{sec}$. Torque analysis of these two designs shows that maximum torque requirement of proposed exoskeleton is only $3.386 \mathrm{mNm}$ whereas motor connected directly to the elbow joint requires $34.5 \mathrm{mNm}$.

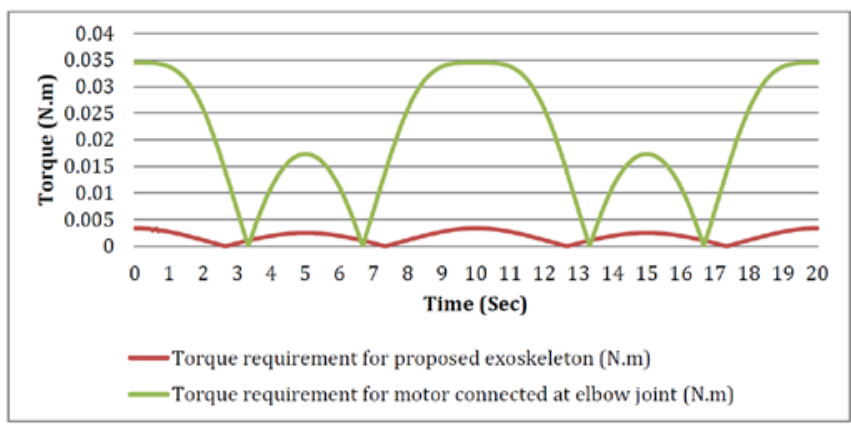

Figure 7. Torque requirements of two models 
The above result shows that the exoskeleton is able to carry out about ten times more weight than joint-axis based motor mechanism. Fig. 8 shows the variation of joint stiffness with the position of slider nut and the elbow joint angle during variable joint stiffness rehabilitation. The figure shows that an incremental increase in the position of the slider results in higher elbow joint stiffness.

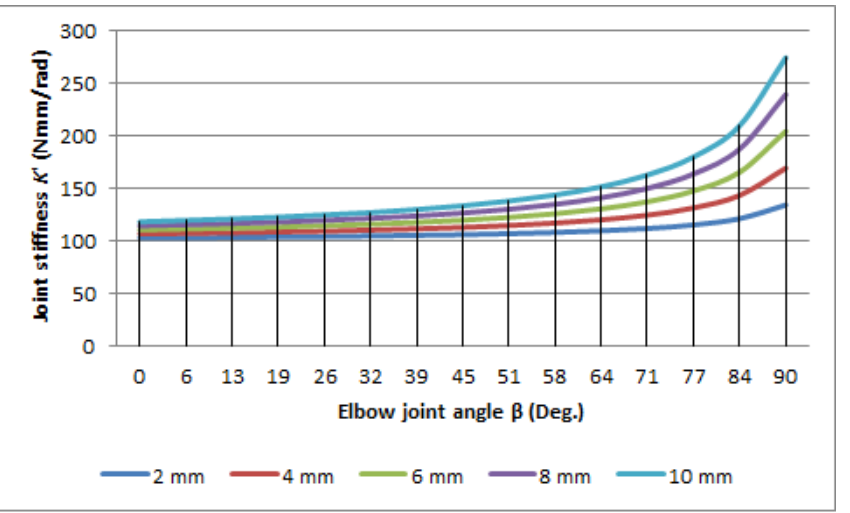

Figure 8. Joint stiffness analysis for different slider positions

\section{CONCLUSION}

The paper presented a new elbow joint exoskeleton which is capable of providing biomechanical advantage over the existing systems. This would provide all necessary training which is essential for different stages of rehabilitation process. The designed exoskeleton is portable which is capable of providing required joint torque without making it a bulky system. The design relies on the hardware based solution which is preferable from a safety point of view. It is able to accommodate actuation complexity with energy efficiency because patients are able to use the exoskeleton for passive as well as variable joint stiffness rehabilitation without needing any power supply. The design offers the advantage of both active and passive rehabilitation in a single system. The stiffness of joint can be changed to improve patient effort during passive rehabilitation.

\section{REFERENCES}

[1] "Occupational therapy after stroke", Stroke Association, 2012. Available:http://www.stroke.org.uk/sites/default/files/F17_Occupation al\%20therapy\%20after\%20stroke.pdf. [Accessed: Sept- 2017].

[2] http://www.gla.ac.uk/news/archiveofnews/2013/may/headline_279079 _en.html

[3] D. Clarke, S. Tyson, H. Rodgers, A. Drummond, R. Palmer, M. Prescott, P. Tyrrell, L. Burton, K. Grenfell, L. Brkic and A. Forster, "Why do patients with stroke not receive the recommended amount of active therapy (ReAcT)? Study protocol for a multisite case study investigation", BMJ Open, vol. 5, no. 8, p. e008443, 2015.

[4] A. Oosterwijk, M. Nieuwenhuis, C. van der Schans and L. Mouton, "Required Shoulder and Elbow Joint Angles in Daily Living Tasks: an Overview (P160)", 2017. [Online]. Available:

http://www.medbc.com/annals/review/vol_28/num_3b/text/vol28n3bp 321.pdf. [Accessed: 26- Apr- 2017].

[5] P. Maciejasz, J. Eschweiler, K. Gerlach-Hahn, A. Jansen-Troy and S. Leonhardt, "A survey on robotic devices for upper limb rehabilitation", Journal of NeuroEngineering and Rehabilitation, vol. 11, no. 1, p. 3, 2014
[6] S. Manna and V. Dubey, "Upper Arm Exoskeletons-What specifications will meet users' acceptability?", in Robotics: New Research, 1st ed., D. Fisher, Ed. New York: Nova Science Publisher, 2017, pp. 123-169.

[7] J. Wolff, C. Parker, J. Borisoff, W. Mortenson and J. Mattie, "A survey of stakeholder perspectives on exoskeleton technology", Journal of NeuroEngineering and Rehabilitation, vol. 11, no. 1, p. 169, 2014.

[8] "The Brunnstrom Stages of Stroke Recovery", Saebo, 2017. [Online]. Available: https://www.saebo.com/the-stages-of-stroke-recovery/. [Accessed: 09- Aug- 2015]

[9] http://titanarm.com/

[10] Z. Song, and S. Guo, "Design Process of Exoskeleton Rehabilitation Device and Implementation of Bilateral Upper Limb Motor Movement”, Journal of Medical and Biological Engineering, vol. 32, no. 5, pp. 323-330, 2011.

[11] http://myomo.com/

[12] Y. Matsumoto, M. Amemiya, D. Kaneishi, Y. Nakashima, M. Seki, T. Ando, Y. Kobayashi, H. Iijima, M. Nagaoka and M. G. Fujie, "Development of an Elbow-Forearm Interlock Joint Mechanism Toward an Exoskeleton for Patients with Essential Tremor", in 2014 IEEE/RSJ International Conference on Intelligent Robots and Systems (IROS 2014), Chicago, IL, USA, 2017, pp. 2055-2062.

[13] J. Zhang, C. Yang, Y. Chen, Y. Zhang and Y. Dong, "Modeling and control of a curved pneumatic muscle actuator for wearable elbow exoskeleton", Mechatronics, vol. 18, no. 8, pp. 448-457, 2008.

[14] N. Vitiello, T. Lenzi, S. Roccella, S. De Rossi, E. Cattin, F. Giovacchini, F. Vecchi and M. Carrozza, "NEUROExos: A Powered Elbow Exoskeleton for Physical Rehabilitation", IEEE Transactions on Robotics, vol. 29, no. 1, pp. 220-235, 2013.

[15] A. Wilkening, H. Stoppler and O. Ivlev, "Adaptive Assistive Control of a Soft Elbow Trainer with Self-Alignment using Pneumatic Bending Joint", in 2015 IEEE International Conference on Rehabilitation Robotics (ICORR), Singapore, 2017, pp. 729-734.

[16] H. WOO, J. Cho, C. Kim and H. Lee, "Development of a 4 DOF Exoskeleton Robot for Elbow and Wrist Rehab", in 2014 14th International Conference on Control, Automation and Systems (ICCAS 2014), Gyeonggi-do, Korea, 2017, pp. 304-306.

[17] D. Popov, I. Gaponov and J. Ryu, "Bidirectional Elbow Exoskeleton Based on Twisted-String Actuators", in 2013 IEEE/RSJ International Conference on Intelligent Robots and Systems (IROS), Tokyo, Japan, 2017, pp. 5853-5858.

[18] T. Haumont, W. Rahman, M. Sample, M. King, C. Church, J. Henley and S. Jayakumar, "Wilmington Robotic Exoskeleton", Journal of Pediatric Orthopaedics, vol. 31, no. 5, pp. e44-e49, 2011. 